\title{
Public impact, prevention, and treatment of cataracts
}

\author{
WU Frances ${ }^{1}$, WANG Sha ${ }^{1}$, ZHU Jie ${ }^{1}$, RUTGARD Jeff ${ }^{1}$, YAN Yong-Bin ${ }^{2}$, ZHANG Kang $^{1}$ \& \\ ZHAO Ling ${ }^{3,4^{*}}$ \\ ${ }^{1}$ Shiley Eye Institute, Department of Ophthalmology and Institute for Genomic Medicine, University of California San Diego, La Jolla, CA \\ 92093, USA; \\ ${ }^{2}$ State Key Laboratory of Membrane Biology, School of Life Sciences, Tsinghua University, Beijing 100084, China; \\ ${ }^{3}$ State Key Laboratory of Ophthalmology, Zhongshan Ophthalmic Center, Sun Yat-sen University, Guangzhou 510060, China; \\ ${ }^{4}$ Institute of Molecular Medicine, Peking University, Beijing 100871, China
}

Received September 11, 2015; accepted September 17, 2015; published online September 30, 2015

Citation: Wu F, Wang S, Zhu J, Rutgard J, Yan YB, Zhang K, Zhao L. Public impact, prevention, and treatment of cataracts. Sci China Life Sci, 2015, 58: 1157-1159, doi: 10.1007/s11427-015-4939-8

The human lens is composed anteriorly of a single layer of epithelial cells, which undergo mitosis and migrate to the lens equator where they differentiate into lens fiber cells [1]. The elongated lens fiber cells express high levels of soluble crystallin proteins that do not undergo turnover and must remain relatively stable over a lifetime. The threedimensional organization of crystallins is crucial for lens transparency and refractive power and therefore visual acuity. The crystallins have some of the highest protein concentrations in the human body and are comprised of two families, the $\alpha$-crystallins and $\beta \gamma$-crystallins. $\alpha$-crystallin acts as a chaperone that sequesters misfolded proteins and prevents widespread protein aggregation. In cataract, the formation of insoluble protein aggregates is believed to overwhelm the native chaperone system [1] (Figure 1). The aggregates scatter light and cause lens opacification, leading to decreased visual acuity and eventually blindness if left untreated.

Cataracts are the leading cause of blindness worldwide and the leading cause of visual impairment in the United States [2]. Annually, millions of people will require surgical treatment for cataract, and this number is only expected to increase as the population ages. The economic, as well as human, costs of the disease are massive. The burden is greatest in the developing world, where most people do not

*Corresponding author (email: Lingzhao1976@126.com) have access to surgical care. In many developing countries, the incidence of new cataract exceeds the rate of growth of surgical services, leading to a backlog of patients who are in need of treatment [3].

Genetic causes of cataract are relatively uncommon and tend to manifest as early onset disease. Congenital cataract is often associated with mutations in crystallin genes. Mutations have also been identified in genes encoding other lens proteins, including membrane proteins, transcription factors, cytoskeletal proteins, and growth factors [4]. Age-related cataract is extremely common in people older than 50 years of age and accounts for the vast majority of this disease. The pathogenesis is thought to be multifactorial, with proteomic analyses demonstrating covalent modifications to wild-type lens proteins in many cases [1]. Molecular and epidemiologic studies have elucidated some of the factors that contribute to the development of age-related cataract. Ultraviolet (UV) radiation and oxidative stress are thought to play particularly important roles. UV radiation from sunlight causes the formation of reactive oxygen species. High levels of glutathione in the lens normally protect lens proteins from oxidation, but they diminish with age, resulting in oxidative damage that in turn drives protein misfolding and aggregation in the development of cataract. In addition, cataract is also a relatively early complication of uncontrolled diabetes. High glucose levels lead to protein glycation, which interferes with the chaperone function of 


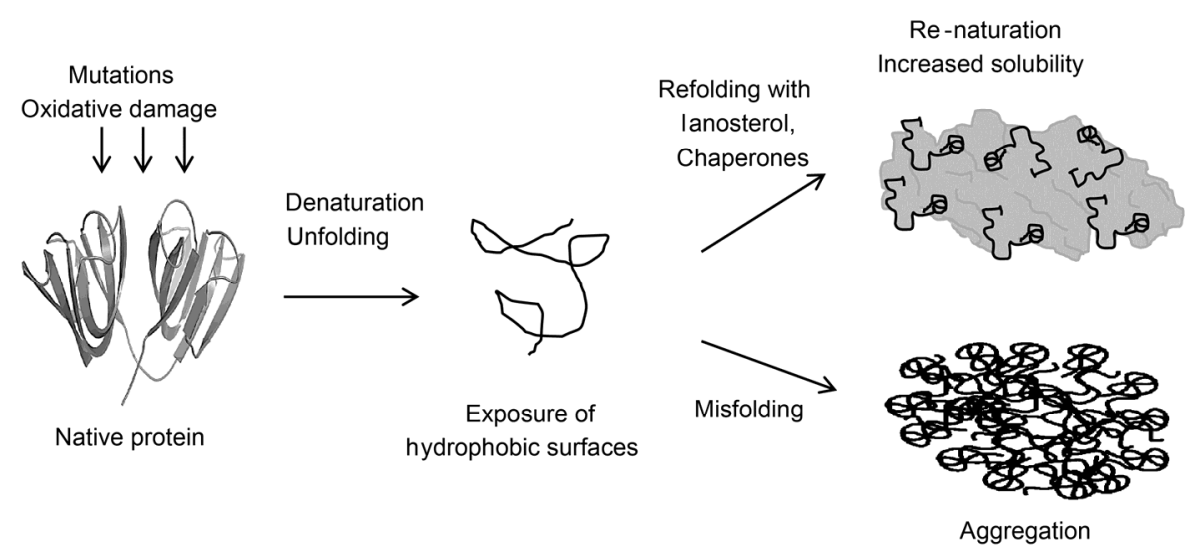

Figure 1 Proposed scheme illustrating a process by which crystallin proteins undergo misfolding and form aggregates, and a potential strategy for reversing aggregation and increasing solubility (modified from reference [1]).

$\alpha$-crystallins and again causes protein misfolding. Other risk factors identified for cataract include cigarette smoking, high alcohol intake, medications, and trauma [2].

The current standard-of-care for visually significant cataracts is surgical management. Cataract extraction with phacoemulsification and intraocular lens (IOL) implantation is the preferred technique. Although this procedure is very effective with a high rate of success, it can be associated with complications such as posterior capsule opacification and necessitating additional procedures to achieve desired visual acuity. In addition, it requires the use of specialized equipment for phacoemulsification. More recently, manual small incision cataract surgery (MSICS) with posterior chamber IOL implantation has been favored in developing countries, as this procedure is relatively rapid and inexpensive [3]. However, access to care in these regions remains limited by cost as well as a lack of facilities and physicians.

Given the limitations posed by the current surgical treatment for cataract in patients in both developed and developing nations, the concept of non-surgical therapy is intriguing. Strategies for cataract prevention using pharmacological therapies have been investigated, but at present there is no FDA-approved treatment. In particular, the role of oxidative damage in the pathogenesis of cataract has fueled interest in antioxidant drugs as a possible therapeutic strategy [5,6]. Synthetic small molecule compounds have been shown to protect lens epithelial cells from oxidative stress in vitro. However, the in vivo delivery of antioxidant drugs to the lens presents several challenges; most importantly, the utility of antioxidants has been hindered by low absorption of topical formulations into the lens nucleus. These antioxidant molecules tend to be either hydrophilic or lipophilic, preventing them from bypassing the lipophilic corneal epithelium or making them poorly water soluble, respectively. Other approaches involving aspirin and aspirin-like drugs and protein stabilizers have also been investigated, but have so far been limited due to undesirable side effects.
A recent study published by Zhao et al. [7] demonstrated that lanosterol is a key molecule in the proper folding of lens proteins. Mutations in lanosterol synthase (LSS), an enzyme in the cholesterol synthesis pathway, were identified in two consanguineous families with congenital cataract. Furthermore, lanosterol had the ability to reduce cataracts in rabbit lenses and in dogs in vivo. Lanosterol is an amphipathic molecule that is hypothesized to intercalate into core hydrophobic areas of protein aggregates and increase their solubility (Figure 1). Topical administration of lanosterol has the advantage of providing a convenient, non-surgical method for prevention and/or treatment of cataracts. It may be useful in patients with early cataract that is not severe enough to warrant surgical intervention, delaying or eliminating the need for surgery in the future. In addition, lanosterol could have utility in treating patients who have contraindications to surgical management of cataract. The impact on the developing world is potentially great, as millions of people with cataract do not have access to surgical care. Blindness and visual impairment contribute to decreased quality of life, accidents, and negative economic consequences stemming from reduced productivity.

The adverse effects of lanosterol treatment will need to be characterized; however, since lanosterol is an endogenous protein, it is expected to have a favorable safety profile. Topical administration should minimize systemic effects while ensuring adequate delivery of drug into the lens material. Because lanosterol is an amphipathic molecule, a topical formulation may have improved bioavailability into the lens nucleus compared to hydrophilic or lipophilic compounds. Nevertheless, there are a number of possible limitations. Lanosterol eye drops may not penetrate dense cataracts, especially those of the nuclear sclerotic subtype. This is because of tight packing of intracellular protein aggregates. Lanosterol may not be effective for hereditary forms of cataract if the three-dimensional structure of lens proteins is highly disrupted, as with cases involving crystallin gene mutations. In addition, cataracts treated with lanos- 
terol are highly likely to recur because the underlying pathogenesis of protein denaturation remains unchanged.

Future directions include determining the specific pharmacokinetics and pharmacodynamics of lanosterol in human lenses, with the goal of formulating an eye drop for eventual treatment of patients with cataracts. Initiation of clinical trials should be feasible within the next few years. Furthermore, lanosterol represents an appealing paradigm for the treatment of cataract and other protein aggregation diseases. These findings support the idea that small molecules may be used to facilitate proper protein folding and prevent or reverse aggregation in neurodegenerative diseases, which collectively account for a significant cause of mobidity and mortality.

1 Moreau KL, King JA. Protein misfolding and aggregation in cataract disease and prospects for prevention. Trends Mol Med, 2012, 18:
273-282

2 Congdon NG, Friedman DS, Lietman T. Important causes of visual impairment in the world today. JAMA, 2003, 290: 2057-2060

3 Chang MA, Congdon NG, Baker SK, Bloem MW, Savage H, Sommer A. The surgical management of cataract: barriers, best practices and outcomes. Int Ophthalmol, 2008, 28: 247-260

4 Shiels A, Hejtmancik JF. Genetics of human cataract. Clin Genet, 2015, 84:120-127

5 Jin H, Randazzo J, Zhang P, Kador PF. Multifunctional antioxidants for the treatment of age-related diseases. J Med Chem, 2008, 53: $1117-1127$

6 Abdelkader H, Alany RG, Pierscionek B. Age-related cataract and drug therapy: opportunities and challenges for topical antioxidant delivery to the lens. J Pharm Pharmacol, 2015, 67: 537-550

7 Zhao L, Chen XJ, Zhu J, Xi YB, Yang X, Hu LD, Ouyang H, Patel SH, Jin X, Lin D, Wu F, Flagg K, Cai H, Li G, Cao G, Lin Y, Chen D, Wen C, Chung C, Wang Y, Qiu A, Yeh E, Wang W, Hu X, Grob S, Abagyan R, Su Z, Tjondro HC, Zhao XJ, Luo H, Hou R, Perry JJ, Gao W, Kozak I, Granet D, Li Y, Sun X, Wang J, Zhang L, Liu Y, Yan YB, Zhang K. Lanosterol reverses protein aggregation in cataracts. Nature, 2015, 523: 607-611

Open Access This article is distributed under the terms of the Creative Commons Attribution License which permits any use, distribution, and reproduction in any medium, provided the original author(s) and source are credited. 